\title{
General Research on Automatic Image Shrinking in the Wireless Capsule Endoscopy
}

\author{
Zhukov Igor \\ National Research Nuclear University “MEPhI”, Kashirskoye highway, 31, Moscow, Russia \\ E-mail: i.zhukov@inbox.ru \\ Fedorov Evgeny \\ Moscow University Hospital №31, Lobachevskogo str., 42, Moscow, Russia \\ E-mail: efedo@mail.ru \\ Mikhaylov Dmitry \\ National Research Nuclear University “MEPhI”, Kashirskoye highway, 31, Moscow, Russia \\ E-mail:mdmitry@mephi.ru \\ Ivanova Ekaterina \\ Moscow University Hospital №31, Lobachevskogo str., 42, Moscow, Russia \\ E-mail: katendo@yandex.ru \\ Kukushkin Alexander \\ National Research Nuclear University "MEPhI”, Kashirskoye highway, 31, Moscow, Russia \\ E-mail: alexanderkuk@ya.ru \\ Starikovski Andrey \\ National Research Nuclear University “MEPhI”, Kashirskoye highway, 31, Moscow, Russia \\ E-mail: userandrew@rambler.ru \\ Tolstaya Anastasia \\ National Research Nuclear University "MEPhI”, Kashirskoye highway, 31, Moscow, Russia \\ E-mail: polynna@yandex.ru
}

\begin{abstract}
In this paper we propose a method of image shrinking without loss of the quality with regard to a modern field in medical research - wireless capsule endoscopy.

The wireless capsule is a small devise with a size of $1,5 \times 2 \mathrm{~cm}$. That means that the memory chip on which the results of the examination of the gastrointestinal tract are stored should also be tiny. The scope of the device imposes strict restrictions on the shrinking scheme that should be taken into consideration.

This article gives a brief overview of existing data shrinking methods and their application possibilities, namely triplets coding of binary combinations, conversion combination MTF (move-to-front) and Rice coding. Taking into consideration the specificity of
\end{abstract}

application the more promising is the third way of image shifting without loss. This method is based on modified shrinking algorithms mentioned above.

According to the carried out experiments the overall scheme of the device was developed. This scheme implements the most efficient method of coding.

The described algorithm allows image shrinking on $20 \%$. That means that endoscopic capsule may work significantly longer.

Index Terms_-image shrinking, capsule, triplets coding, Rice coding, gastrointestinal tract, scheme.

\section{PAPER STRUCTURE}

The paper is organized in the following way: 
- An introduction telling about wireless capsule endoscopy.

- Explanation of the needs of image shrinking.

- Description of existing methods of image compression, namely triplets coding of binary combinations, conversion combination MTF (move-to-front) and Rice coding.

- The description of the proposed algorithm in details with a help of the scheme.

- Results in figures.

- Conclusion.

\section{INTRODUCTION}

Wireless capsule endoscopy is a modern noninvasive method of the gastrointestinal tract examination.

Capsule endoscopy has provided clinicians with the non-invasive ability to detect abnormal changes of the gastrointestinal tract (generally in small bowel) often missed by other techniques. [1], [2].

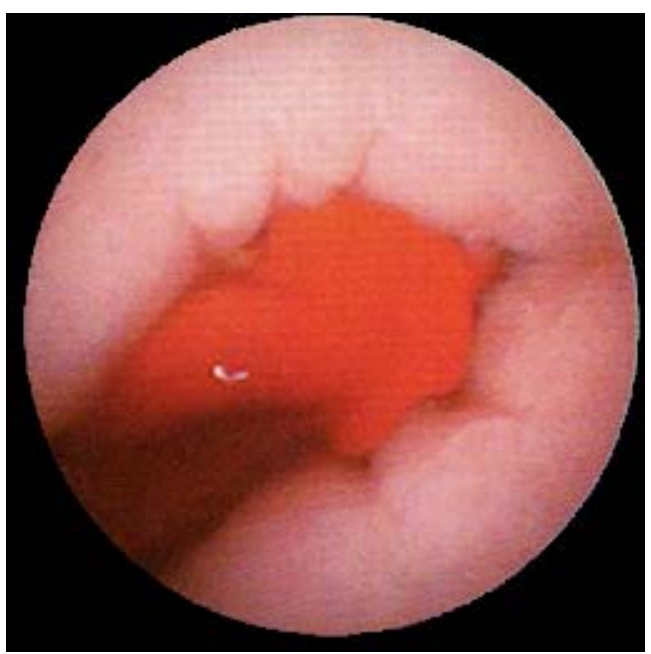

Figure. 1. Image of the gastrointestinal tract in the wireless capsule endoscopy.

The possibility of capsule to make an automatic analysis is now of great interest because of its potential in recognition of pathologies in any part of gastroenterological tract. However, this method of examination of the gastrointestinal tract requires a lot of memory for data storage [3].

This article is devoted to image shrinking method without loss of the quality with regard to a modern field in medical research - wireless capsule endoscopy.

Person swallows a tiny capsule that takes pictures of his digestive tract. In the diagnosis of the gastrointestinal tract wireless capsule travels taking two images per second and by the end of the examination there are more than 57,000 images (as shown in Fig. 1). After that images are transmitted to a desktop computer for further processing. In the end this images are analyzed be a physician [4]

One of the most important issues of the wireless endoscopy system is providing sufficient capacity of the transmitter, the power supply duration and the equipment size. The decrease of the transmitted information will increase image acquisition frequency reducing energy consumption. As a result endoscopic capsule may work significantly longer. Thus, there is a problem of image shrinking inside the capsule in order to get more data for analysis without increasing the size of the device.

\section{SHRINKING SCHEMES}

Data from the camera of the wireless capsule are transmitted to the memory on the principle of FIFO (First In - First Out) [5]. From the memory the array comes on the shrinking chip over a single-bit port. The color image pixel is determined by three components of the scheme RGB (Red, Green, Blue). Then the data are shrunk and sent over an 8-bit port to the transmitter. Capsule transmitter sends code words to the external receiver which decodes the image.

The scope of the device imposes strict restrictions on the shrinking scheme [6]:

- Low power consumption - capsule works on battery and high energy consumption will reduce the time of work.

- Memory array is updated twice a second - the time of shrinking should not exceed this limit.

- The size of capsule is $1.5-2 \mathrm{~cm}$. It imposes a limit on the chip size. The best option will be a chip $5 \times 5 \mathrm{~mm}$.

According to the requirement of the device for data shrinking without the loss the following algorithms were chosen:

- $\quad$ Triplets coding of binary combinations (TC);

- Conversion combination MTF (move-to-front) [7], and Rice coding. 


\section{A. Triplets coding of binary combinations}

Triplets coding of binary combinations refers to the universal coding. In the universal coding statistical redundancy in the sequence obtained after coding tends to zero with the length increasing of the blocks into which the original binary sequence is divided.

This method is designed to shrink the binary data in an unknown statistics of the message source. The method is used when it is needed to restore exactly the original binary sequence and data shrinking is unacceptable due to information loss [8], [12], [13], [14].

The original sequence of binary data is divided into blocks of $\boldsymbol{n}$ bits. For each block three parameters are determined:

- The number of units in the block - $k$;

- The sum number of their positions - $s$;

- The number of this particular combination in the corresponding class (element of the intersection of the multitudes $K$ and $S)-b(n, k, s)$.

It is proved that the redundancy $R_{n}$ in the dataflow output tends to zero with the length increasing of the original blocks $\boldsymbol{n}$. It means that coding is asymptotically optimal:

$$
\begin{aligned}
& \lim _{n}=0 \text {, where } \\
& n \rightarrow \infty \\
& R_{n-} \text { coding redundancy: } \\
& R_{n}=\sup R_{n}(p), \\
& \text { where } o<p<1 \\
& R_{n}(p)=n_{c p} / n-H(p),
\end{aligned}
$$

where $n_{c p}$ is average length of code word,

$H(p)=-\left(p \log _{2} p+q \log _{2} q\right)-$ entropy of the source.

The number of each code combination is calculated by the formula:

$b(n, k, s)=R\left(i_{k}-1, K, i_{k}+i_{k-1}+\ldots+i_{1}\right)+R\left(i_{k-1}-1, K-1, i_{k-}\right.$ $\left.{ }_{1}+i_{k-2} \ldots+i_{1}\right)+\ldots+R\left(i_{2}-1,2, i_{2}+i_{1}\right)=$

$=\sum_{j=2}^{k} R\left(i_{j}-1, J, \sum_{m=1}^{j} i_{m}\right)$, where $i$ is the number of the unit position in the original block [8].

For the complexity reduction of the implementation it makes sense to adapt the TC i.e. to add an extra bit in code word that contains an indication to encode the information or not according to the number of units in the input block.

\section{B. Conversion combination MTF and Rice coding}

MTF is a conversion to encode the data (usually a byte stream) designed to improve the performance of entropy coding. The basic idea is to replace each input symbol by its number in a special stack (register of recently used symbols) [9].

The sequences of identical symbols will be replaced by the original algorithm (starting with the second symbol) into a sequence of zeros. If the symbol does not appear in the input sequence for a long time it will be equal to a large number. The input symbols are replaced by a sequence of integers. If the input data have a lot of local correlations they are better for shifting that the original ones [10], [11].

Rice code is a family of binary prefix codes of natural numbers and zero representation. Codes differ by a single parameter $k$ - number of bits allocated to the number mantissa. The method of encoding and decoding depends on the setting. So it should be known either by the transmitter or by the receiver.

The encoded number in binary notation is divided into two parts: $k$ of the least significant bits and the rest of the $\mathrm{k}^{\text {th }}$ and high order. The high order part is encoded by the unary code and the least significant is recorded after the encoded high order part. For an $n$-block this code is given in $(n / m+k+1)$ bits, where $m=2^{k}$ is a selected number that is a power of two [10].

As a result of simulation and testing of the developed devices the output file size and the duration of data processing were investigated. As TC parameters presence / absence of adaptation were used. In the presence of adaptation different minimum number of 
units was used. The test results were compiled in Tables 1 and 2 in which the following notation were agreed:

$\mathrm{k}_{\text {min }}$ - minimum value.

$\mathrm{C}_{\text {sh }}$ - the data shrinking coefficient.

$\mathrm{t}$ - number of tacts (millions).

KB - kilobyte.

TABLE.1 DEPENDENCE OF SHIFTING COEFFICIENT TC FROM VARIOUS PARAMETERS

\begin{tabular}{|c|c|c|c|c|c|}
\hline $\mathrm{n}$ & $\begin{array}{l}\mathrm{t}, \mathrm{mil} \\
\text { tacts }\end{array}$ & $\begin{array}{l}\text { Size, } \\
\text { KB } \\
\end{array}$ & Adaptation & $\begin{array}{l}\mathrm{k} \\
\min \end{array}$ & $\mathrm{C}_{\mathrm{sh}}$ \\
\hline 8 & 30,5 & 325 & no & - & 0,94 \\
\hline 16 & - & 213 & no & - & 1,44 \\
\hline 24 & - & 160 & no & - & 1,92 \\
\hline \multirow{5}{*}{24} & 3,3 & 273 & yes & 2 & 1,12 \\
\hline & 3,7 & 267 & yes & 3 & 1,14 \\
\hline & 5,2 & 267 & yes & 4 & 1,14 \\
\hline & 11,2 & 267 & yes & 5 & 1,14 \\
\hline & 45,4 & 267 & yes & 6 & 1,14 \\
\hline \multirow{5}{*}{32} & 3,2 & 268 & yes & 2 & 1,15 \\
\hline & 3,5 & 264 & yes & 3 & 1,16 \\
\hline & 5,2 & 262 & yes & 4 & 1,17 \\
\hline & 15,5 & 260 & yes & 5 & 1,18 \\
\hline & 60,1 & 259 & yes & 6 & 1,19 \\
\hline \multirow{4}{*}{48} & 3,2 & 259 & yes & 3 & 1,19 \\
\hline & 3,6 & 255 & yes & 4 & 1,20 \\
\hline & 5,9 & 251 & yes & 5 & 1,22 \\
\hline & 15,35 & 249 & yes & 6 & 1,23 \\
\hline 64 & 80,5 & 254 & yes & 5 & 1,20 \\
\hline 128 & 8,4 & 255 & yes & 4 & 1,20 \\
\hline 256 & 12,6 & 256 & yes & 4 & 1,19 \\
\hline 512 & 24,1 & 259 & yes & 4 & 1,18 \\
\hline
\end{tabular}

TABLE.2 DEPENDENCE OF SHIFTING COEFFICIENT IN RICE CODING FROM VARIOUS PARAMETERS

\begin{tabular}{|l|l|l|l|l|l|}
\hline $\mathrm{k}$ & 2 & 3 & 4 & 5 & 6 \\
\hline $\mathrm{C}_{\text {sh }}$ & 0,50 & 0,78 & 0,98 & 1,11 & 1,09 \\
\hline Size, KB & 612 & 392 & 302 & 275 & 281 \\
\hline $\mathrm{t}$, mil tacts & 1,8 & \multicolumn{5}{|l|}{} \\
\hline MTF & no \\
\hline $\mathrm{k}$ & 2 & 3 & 4 & 5 & 6 \\
\hline $\mathrm{C}_{\text {sh }}$ & 0,73 & 1,04 & 1,21 & 1,21 & 1,12 \\
\hline Size, KB & 415 & 295 & 254 & 254 & 274 \\
\hline t, mil tacts & 23 & & \\
\hline MTF & yes
\end{tabular}

As a result $\mathrm{TC}$ shrinking method with the parameters mentioned above was chosen for the following reasons:
- TC processes an input block for less tacts.

- TC has a higher shrinking coefficient.

- For the TC implementation with the block length of 48 bits less memory is required.

The following variants of TC implementation are possible:

- The programmatic approach with the help of computer.

- Hardware-software method using single-board computer or computer on a single chip.

- Development of specialized devices on basis of the original chip using flash technology.

\section{The proposed method of image shrinking}

Taking into consideration the specificity of application the more promising is the third way of image shifting without loss. The proposed algorithm of image shrinking is based on above mentioned methods but with a number of modifications and adding. As a result of the procedure the images of that are transferred to a capsule are shrunk on $20 \%$. The results are shown in Fig. 2.

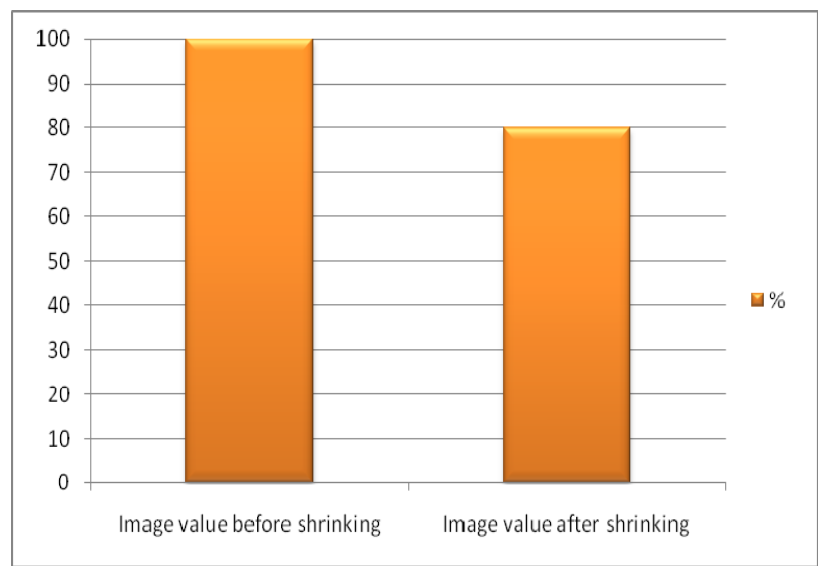

Figure 2. The results of image shrinking process.

The chip input receives binary sequence with the statistical redundancy and removed. From the output the sequence deprived of redundancy is received, i.e. data shifting is provided.

In Fig. 3 an algorithm implementing TC is shown. The following notations are agreed:

- $\quad k-$ the number of units in the original block;

- $s$ - the sum number of units positions in the original block; 
- $\quad b$ - the number of each particular code combination for fixed values of $k$ and $s$;

- $\quad N, K, S-$ the service parameters for the $b$ number calculation by the formula (2).

- $\quad r(n, k, s)$ - the number of binary combinations with $k$ units and the sum of their position $s$ in the $n$ - bit block;

- $A(I)$ - the one-dimensional array of bits of the analyzed input word;

- $\quad I$ - the current service index of units positions numbers.

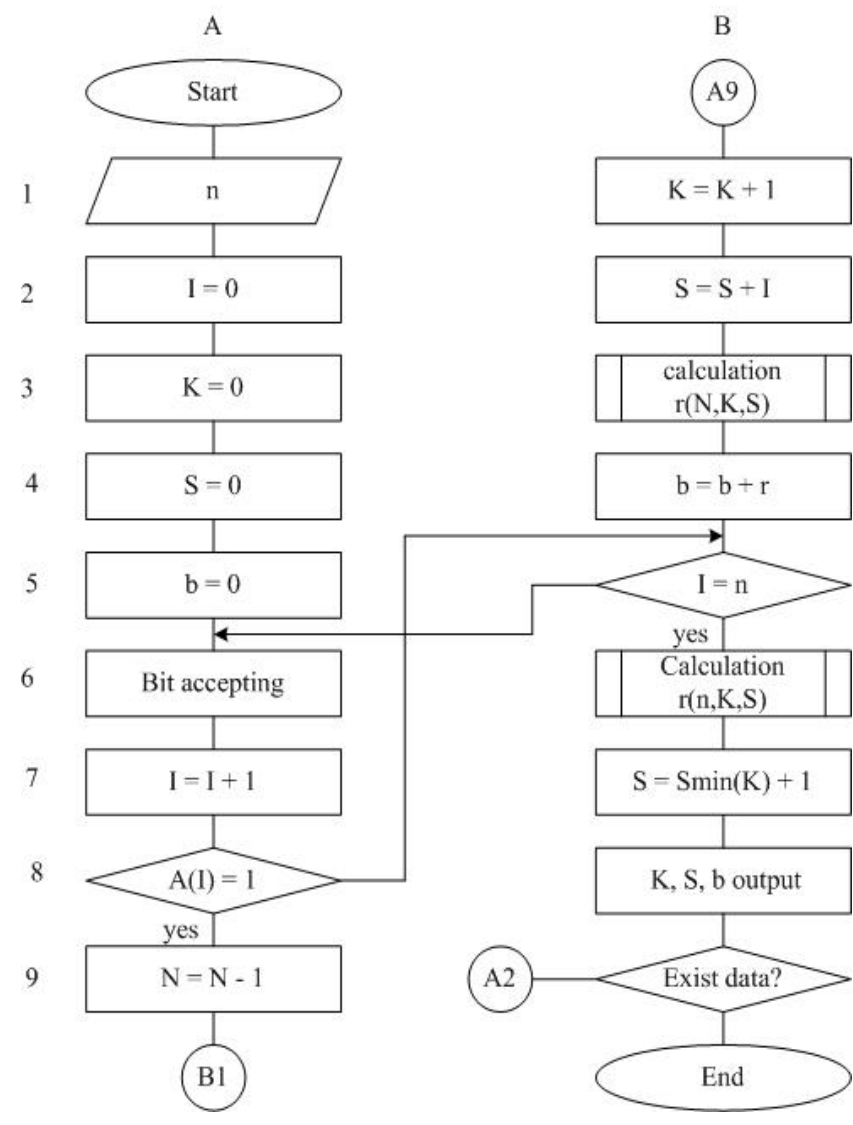

Figure. 3. Encoding algorithm.

The $\mathrm{n}$ value is considered to be given specified. It is the block length into which the original sequence of binary symbols is divided (block A1).

$\mathrm{I}$ is the number of processed n-block; $\mathrm{k}$ is current value of the number of units in the block; $\mathrm{S}$ is the sum of the numbers of their positions; $b$ is the number calculated by the formula (2); A (I) is the contents of the $\mathrm{I}^{\text {th }}$ bit of n-block.

In blocks A2, A3, A4, A5 codeword parameters have initial values.

From the output of the channel the binary data are received. In block A6 the following bit of the sequence is transmitted. In block A7 the value of I is incremented by the unit.

In block A8 it is determined whether contents of the $\mathrm{I}^{\text {th }}$ bit of n-block is equal to unit. If "Yes" the corresponding items of formula (2) are calculated in the codeword (blocks A9, B1, 2, 3). In block B4 the calculated item is added to the current value of the number $b$.

If in block A8 it turns out that the contents of the $\mathrm{I}^{\text {th }}$ bit of n-block is equal to zero the process moves to block B5. It is revealed if all bits of n-block are checked. If "No" the process moves to block A6. If "Yes" in block B6 the sum of units' positions of the original block is modified. The original value of $\mathrm{S}$ is replaced by its number.

In block B7 the coefficient needed to determine the number of bits required for the transmitted codeword number is calculated. In block B8 a generated codeword is provided. If the channel continues to receive data the process moves to block A2.

\section{CONCLUSION}

The wireless capsule endoscopy still faces the problem of image shrinking inside the capsule in order to get more data for analysis without increasing the size of the device.

The suggested method shrinks images received from an endoscopic capsule on $20 \%$ without any loss. The method is based on the research of existing methods of image shrinking advantages of which are used in proposed algorithm.

This method is complied with all the technical requirements made by the wireless capsule endoscope.

The shrinking of the transmitted information will increase image acquisition frequency reducing energy consumption. As a result endoscopic capsule may work for a longer period of time.

The proposed method of automatic image shrinking in the wireless capsule endoscopy will be 
improved and modified for better information compression.

In conjunction with the software installed on the receiver that automatically selects the images suitable for the research the developed method provides exhaustive information about gastrointestinal tract in a form suitable for analysis.

\section{ACKNOWLEDGMENT}

We would like to thank $\mathrm{PhD}$ in medical sciences, doctor of higher category of The Clinical Hospital № 85, Moscow, Russia Gubaidulina Landish Ildousovna for her inestimable contribution in this study.

\section{REFERENCES}

[1]. Mergener K., Ponchon T., Gralnek I. [et al.] Literature review and recommendations for clinical application of small-bowel capsule endoscopy, based on a panel discussion by international experts: consensus statements for small-bowel capsule endoscopy 2006/2007 // Endoscopy. - 2007. Vol.39. - P.895-909.

[2]. Friedman S. Comparison of capsule endoscopy to other modalities in small bowel. Gastrointest Endosc Clin N Am 2004; 14: 51-60.

[3]. Iddan G., Swain P. History and development of capsule endoscopy. Gastrointest Endosc Clin N Am 2004; 14: 1-9.

[4]. Eliakim R, Fischer D, Suissa A, Yassin K, Katz D, Migdal M, Guttman N. Wireless capsule video endoscopy is a superior diagnostic tool compared to barium folloew through and CT in patients with suspectrd Crohn's disease. Europ J Gastroenterol Hepatol 2003; 15: 363-367.

[5]. Expert Verilog, SystemVerilog \& Synthesis Training. Simulation and Synthesis Techniques for Asynchronous FIFO Design with Asynchronous Pointer Comparisons. SNUG-2002.

[6]. Hartmann D. et al. Capsule Endoscopy, Technical impact, benefits end limitations. Langenbek $\mathrm{s}$ Arhives of Surgery 2004; 389: 3: 225-233.

[7]. J. L. Bentley, D. D. Sleator, R. E. Tarjan, V. K. Wei, A Locally Adaptive Data Compression Scheme, Communications of the ACM-Vol. 29, No. 4, 1986.

[8]. Aleksandrovich A.E., Yadykin I.M., Shurygin V.A. The method of universal coding of binary data. Journal "Problems of Radio Electronics", Volume 2, Moscow, 2011, p.94-115.
[9]. Arnavut, Z.; Dept. of Math. \& Comput. Sci., State. Data Compression Conference, 2000. Proceedings. DCC 2000. Univ. of New York, Fredonia, NY // 2000. 193 202.

[10]. V. Sergeenko, V.V. Barinov. Data, speech, sound and images shrinking in telecommunication systems. RadioSoft / 2010.

[11]. Vatolin D., Ratushniak A., Smirnov M., Yukin V. Methods of data shrinking. Archives arragement, image and video shrinking. - Moscow: DialogueNRNU MEPhI, 2003. - 384.

[12]. V.A. Shurygin. Binary data shrinking in an unknown statistics source. - M.: 1985.

[13]. V.A. Shurygin. Universal Coding - lossless shrinking. Scientific works "Telecommunications and New Information Technologies in Education". Moscow: NRNU MEPhI, 2010.

[14]. Robert P. Dobrow and James Allen Fill. The moveto-front rule for self-organizing lists with Markov dependent requests. The Johns Hopkins University. 2005. 